\title{
Avaliação de minerais em plantas medicinais amazônicas
}

\author{
Alexsandro Sozar Martins, ${ }^{* 1}$ Cláudio Nahum Alves, ${ }^{2}$ Osmar A. Lameira, ${ }^{3}$ \\ Alberdan S. Santos, ${ }^{2}$ Regina Celi Sarkis Müller ${ }^{1}$ \\ ${ }^{1}$ Laboratório de Controle de Qualidade e Meio Ambiente, Universidade Federal do Pará, \\ Rua Augusto Correa, 01, Guamá, 66750-110 Belém-PA, Brasil \\ ${ }^{2}$ Laboratório de Investigação Sistemática em Biotecnologia, Universidade Federal do Pará, \\ Rua Augusto Correa, 01, Guamá, 66750-110 Belém-PA, Brasil \\ ${ }^{3}$ Laboratório de Biotecnologia, Embrapa Amazônia Oriental, Tv. Enéas Pinheiro, s/n. Marco, \\ 66095-100 Belém-PA, Brasil
}

\begin{abstract}
RESUMO: Amazônia brasileira oferece um apreciável potencial de plantas com propriedades terapêuticas, embora a maioria seja pouco conhecida. Dessa forma, com o objetivo de verificar a potencialidade nutricional de ervas medicinais, determinou-se a concentração de $\mathrm{Ca}, \mathrm{Mg}, \mathrm{Fe}, \mathrm{Cu}$ e Zn nas folhas e nos chás das espécies: Piper callosum Ruiz \& Pav., Piperaceae, Mikania lindleyana DC., Asteraceae e Arrabidaea chica (Humb. \& Bonpl.) B. Verl., Bignoniaceae. As amostras de plantas depois de terem sido processadas, foram submetidas a digestão e em seguida realizada as leituras dos metais em um espectrofotômetro de absorção atômica. Para o chá de Arrabidaea chica foram detectados teores de Ca (6955 a $20058 \mathrm{mg} / \mathrm{L}), \mathrm{Mg}(2390$ a $3094 \mathrm{mg} / \mathrm{L})$ e Fe (40 a 61 $\mathrm{mg} / \mathrm{L})$. Para o chá de Mikania lindleyana além da presença de altos valores de Ca (17722 a 22336 $\mathrm{mg} / \mathrm{L}), \mathrm{Mg}(4531$ a $9370 \mathrm{mg} / \mathrm{L})$ e Fe $(20$ a $87 \mathrm{mg} / \mathrm{L})$ foram encontrados de 7 a $16 \mathrm{mg} / \mathrm{L}$ de $\mathrm{Cu}$ e 9 a $41 \mathrm{mg} / \mathrm{L}$ de Zn. O chá do Piper callosum apresentou em média 2036 a $4344 \mathrm{mg} / \mathrm{L}$ de Ca, 618 a $4023 \mathrm{mg} / \mathrm{L}$ de $\mathrm{Mg}$ e 39 a $60 \mathrm{mg} / \mathrm{L}$ de Fe. Comparando-se os resultados dos minerais com os valores recomendados pela Organização Mundial da Saúde, conclui-se que os metais presentes nos chás das plantas poderiam contribuir na complementação das dietas alimentares das pessoas que as utilizam.
\end{abstract}

Unitermos: Mikania lindleyan, Arrabidaea chica, Piper callosum, fitoterapia, análise mineral.

\begin{abstract}
Minerals evaluation in Amazonian medicinal plants. The Amazonian Brazilian offers an appreciable potential of plants with therapeutic properties, although most are little known. In this way, with the objective of verifying the potentiality nutritional of medicinal herbs, a work was developed to determine the concentration of $\mathrm{Ca}, \mathrm{Mg}, \mathrm{Fe}, \mathrm{Cu}$ and $\mathrm{Zn}$ in the leaves and in the teas of these species: Piper callosum Ruiz \& Pav., Piperaceae, Mikania lindleyana DC., Asteraceae e Arrabidaea chica (Humb. \& Bonpl.) B. Verl., Bignoniaceae. After the plants samples have been processed, they were submitted to digestion and soon afterwards the metals were analyzed in an spectrophotometer of Atomic Absorption. The results showed the follow yields: for the tea of Arrabidaea chica Ca were detected (6955 to $20058 \mathrm{mg} / \mathrm{L}$ ), $\mathrm{Mg}$ (2390 to $3094 \mathrm{mg} / \mathrm{L}$ ) and Fe (40 to $61 \mathrm{mg} / \mathrm{L})$. For the tea of Mikania lindleyana besides the presence of high values of $\mathrm{Ca}$ (17722 to $22336 \mathrm{mg} / \mathrm{L}$ ), $\mathrm{Mg}$ (4531 to $9370 \mathrm{mg} / \mathrm{L}$ ) and Fe (20 to $87 \mathrm{mg} / \mathrm{L})$ they were found from 7 to $16 \mathrm{mg} / \mathrm{L}$ of $\mathrm{Cu}$ and 9 to $41 \mathrm{mg} / \mathrm{L}$ of $\mathrm{Zn}$. The tea of the Piper callosum presented 2036 to $4344 \mathrm{mg} / \mathrm{L}$ of Ca, 618 to $4023 \mathrm{mg} / \mathrm{L}$ of $\mathrm{Mg}$ and 39 to $60 \mathrm{mg} / \mathrm{L}$ of Fe. Being compared the results of the minerals with the values recommended by the Health World Organization, is possible that the present metals in the teas of the plants could contribute in the complementation of the people's alimentary diets that use these medicinal plants.
\end{abstract}

Keywords: Mikania lindleyan, Arrabidaea chica, Piper callosum, phytotherapic, mineral analysis.

\section{INTRODUÇÃO}

Amazônia brasileira oferece um apreciável potencial de plantas com propriedades terapêuticas, embora a maioria seja pouco conhecida. Muitas destas espécies são empregadas comumente pela população apenas com base na informação popular (Lopes et al., 1996; Almeida et al., 2002), sem o conhecimento dos seus princípios ativos, o que dificulta uma avaliação de suas potencialidades medicinais e o seu aproveitamento econômico. (Berg, 1993; Maciel et al., 2002).

A espécie Arrabidaea chica (Humb. \& Bonpl.) B. 
Verl., conhecida popularmente como pariri, é utilizada na fitoterapia popular tanto de maneira externa quanto interna como antiinflamatório. O uso tópico, na forma de banhos, é preconizado nos casos de ferimentos, impigens, lavandose a área afetada até a cura. Para o uso interno é indicado o chá das folhas em forma de infusão, que é ingerido de forma continuada, substituindo a água na dieta alimentar normal, utilizada em casos de anemias, inflamações uterinas, diarréias sanguíneas, leucemias, hemorragias, diurético, albumina elevada, hepatites e nervosismo (Lorenzi \& Matos, 2002; Martins et al. 2005).

O elixir paregórico (Piper callosum Ruiz \& Pav.) é usado na medicina popular através de chás das folhas na forma de infusão para tratar cólicas menstruais e intestinais, diarréia, dismenorréia, dor de diversas origens, principalmente do aparelho digestivo, dor reumática e muscular, hemorragia local, náusea, picadas de mosquito, problemas digestivos, como dor de estômago, diarréia e reumatismo (Berg, 1993).

A espécie Mikania lindleyana DC., conhecida como sucurijú, é caracterizada por ser trepadeira sobre árvores na beira do igapó, por possuir flor branca e cheirosa É utilizada empiricamente para tratar dermatose, hepatite, inflamação, úlcera gástrica crônica e varicose. Também é utilizada como diurético, analgésico e anti-hipertensivo. É administrada de forma oral através de chás das folhas (Berg, 1993; Martins et al. 2005).

Muitos autores já demonstraram interesse em estudar as possibilidades de suplementação alimentar de minerais com plantas medicinais (Almeida et al., 2002; Lopes et al., 2002; Andrade et al., 2005; Delaporte et al., 2005). Os minerais são indispensáveis na alimentação humana, no entanto, muitas vezes há um consumo inadequado desses componentes na dieta nutricional de vários grupos de pessoas, principalmente as de baixa renda (Flores et al., 1998).

Os sais minerais realizam diversas funções especificas no corpo humano estando relacionados com o bom desempenho do metabolismo de enzimas, as quais são responsáveis pela manutenção da saúde do organismo (Harper \& Mayes, 1982; Franco, 1998; Duarte \& Pasqual, 2000). Entre os macronutrientes o cálcio e o magnésio participam da constituição da estrutura óssea, dentes e tecidos, e são necessários para ativação de enzimas que participam do processo de digestão dos alimentos, assim como, na permeabilidade seletiva da membrana plasmática (FAO/WHO, 1998; Pinto et al., 1999; Lopes et al., 2002).

O zinco, ferro e cobre são necessários em pequenas quantidades pelo organismo, sendo por isso chamados de micronutrientes, no entanto, também indispensáveis na alimentação. (Favier, 1991; Krause \& Mahan, 1991). O zinco é um elemento importante para o crescimento, reprodução, cicatrização de ferimentos, ativação de reações catalisadas por enzimas antioxidantes, além de funções imunológicas (WHO, 1996). O ferro por fazer parte de moléculas do sangue como hemoglobina e mioglobina é um nutriente indispensável no transporte de oxigênio e respiração celular (Burton, 1979; Machado et al., 2006). Já o cobre é constituinte de enzimas como a $C$-oxidase e monoaminooxidase, além de contribuir para manutenção do sistema nervoso central e atividade cardiovascular (Burton, 1979; Lehninger et al., 1998).

O uso contínuo e compulsório de espécies vegetais no combate ou alívio de doenças motiva a determinação da composição inorgânica dessas ervas. Dessa forma, determinou-se o conteúdo de minerais presentes em chás e folhas das espécies elixir paregórico (Piper callosum), sucurijú (Mikania lindleyana) e pariri (Arrabidaea chica), com objetivo de verificação das potencialidades nutricionais de cada espécie.

\section{MATERIAL E MÉTODOS}

\section{Amostragem}

Foram utilizadas cinco amostras de elixirparegórico (Piper callosum), cinco de sucurijú (Mikania lindleyana) e cinco de pariri (Arrabidaea chica), as quais foram amostradas de dois canteiros para cada espécie. As amostras foram coletadas no $1^{\circ}$ semestre de 2005 em três localidades: horto de plantas medicinais da EMBRAPA Amazônia Oriental (Belém-PA), horto de plantas medicinais da ALBRAS (Barcarena-PA) e na feira livre do Ver-o-peso (Belém-PA).

O solo das plantas coletadas no horto da EMBRAPA foi considerado como padrão de referência para as outras localidades por ser administrado por agrônomos funcionários da empresa. Este solo recebe tratamento com adubação orgânica composta de esterco bovino e restos de plantas. Já o solo do horto da ALBRAS, localizado na comunidade Vai-quem-quer, na PA $483 \mathrm{~km}$ 10 , é cultivado por agricultores que recebem incentivos da ALBRÁS através do Projeto de Agricultura Familiar Mecanizada (PAFAM). Este solo é tratado com adubação de caroços de açaí e restos de alimentos. O cultivo das plantas medicinais nos hortos da EMBRAPA e ALBRAS é feito em canteiros de dimensões de $80 \times 80 \mathrm{~cm}$, sob proteção de tela de sombrite a 70\%. As amostras compradas na feira livre do Ver-o-peso foram incluídas nesse trabalho para se ter idéia das ervas vendidas para população, cuja procedência é bastante variada.

A identificação das espécies foi realizada por $\mathrm{R}$. K. S. Sakurai e S. T. Rodrigues. e as exsicatas encontramse no Laboratório de Botânica da EMBRAPA Amazônia Oriental.

\section{Preparo das amostras e determinação dos teores dos minerais}

Os materiais vegetais coletados, após passarem por um processo de seleção e limpeza, foram pesados e secos em uma estufa (Fanem 3155E) até peso constante. 
Trituraram-se as folhas obtendo-se um pó fino. Transferiuse $200 \mathrm{mg}$ do pó de folhas para um tubo digestor, adicionou-se $4 \mathrm{~mL}$ de ácido nítrico concentrado e $1 \mathrm{~mL}$ de ácido perclórico concentrado. Em seguida colocaram-se os tubos com as amostras em um do bloco digestor (Tecnal 040125) a $180^{\circ} \mathrm{C}$ até a completa digestão. Transferiu-se o extrato obtido para um balão volumétrico de $50 \mathrm{~mL}$, completando-se o volume com água ultrapura (Silva, 1999).

Para o preparo dos chás, pesou-se $250 \mathrm{mg}$ do pó das folhas e adicionou-se $50 \mathrm{~mL}$ de água ultrapura fervente. Logo em seguida filtrou-se o extrato (Lameira et al., 2004).
A determinação de $\mathrm{Ca}, \mathrm{Mg}, \mathrm{Fe}, \mathrm{Cu}$ e $\mathrm{Zn}$ foi realizada em um espectrofotômetro de absorção atômica (modelo 220-Varian). As curvas analíticas foram preparadas a partir de soluções estoques de $1000 \mathrm{mg} / \mathrm{L}$ (SpecSol), as quais foram diluídas nas concentrações referentes à curva de cada elemento. Para a leitura de $\mathrm{Ca}$ e $\mathrm{Mg}$ transferiu-se $1 \mathrm{~mL}$ da amostra para um balão de $50 \mathrm{~mL}$, adicionou-se $10 \mathrm{~mL}$ de uma solução a $5 \%$ de $\mathrm{La}\left(\mathrm{NO}_{3}\right)_{3} \cdot 10 \mathrm{H}_{2} \mathrm{O}$ (para evitar interferências de ionização do cálcio), e completou-se o balão com água ultrapura. As determinações de $\mathrm{Fe}, \mathrm{Cu}$ e $\mathrm{Zn}$ foram feitas nas amostras sem diluições. As condições instrumentais para as análises dos minerais estão ilustradas na Tabela 1.

Tabela 1. Condições instrumentais para as análises dos minerais

\begin{tabular}{ccccccc}
\hline Elemento & Equação da reta & Limite de deteç̧ão $(\mathrm{mg} / \mathrm{L})$ & $\mathrm{R}^{2}$ & Fenda $(\mathrm{nm})$ & Curva analítica $(\mathrm{mg} / \mathrm{L})$ & $\lambda(\mathrm{nm})$ \\
\hline $\mathrm{Ca}$ & $\mathrm{y}=0,002 \mathrm{x}+0,001$ & 0,059 & 0,998 & 0,5 & $1--4$ & 422,7 \\
$\mathrm{Mg}$ & $\mathrm{y}=0,031 \mathrm{x}+0,004$ & 0,005 & 0,997 & 0,5 & $0,10-0,40$ & 285,2 \\
$\mathrm{Fe}$ & $\mathrm{y}=0,0032 \mathrm{x}-0,003$ & 0,130 & 0,998 & 0,2 & $2-8$ & 248,3 \\
$\mathrm{Cu}$ & $\mathrm{y}=0,005 \mathrm{x}-0,004$ & 0,040 & 0,997 & 0,5 & $1-4,90$ & 324,8 \\
$\mathrm{Zn}$ & $\mathrm{y}=0,017 \mathrm{x}+0,004$ & 0,020 & 0,997 & 1,0 & $0,4-1,40$ & 213,9 \\
\hline
\end{tabular}

\section{RESULTADOS E DISCUSSÃO}

\section{Determinação dos teores dos minerais nas folhas e chás das plantas}

As Tabelas 2 e 3 mostram os resultados da determinação dos minerais $\mathrm{Ca}, \mathrm{Mg}, \mathrm{Fe}, \mathrm{Cu}$ e $\mathrm{Zn}$ nas folhas e chás das plantas analisadas. As amostras foram classificadas de acordo com o local de procedência. Os valores listados nas Tabelas correspondem às médias dos resultados obtidos de cinco replicatas para as análises das folhas e em triplicata para os chás, ao lado dessas medidas estão os desvios padrões das análises..

Observa-se na Tabela 2 que a espécie pariri apresentou nas folhas elevados teores de cálcio (15045 a $20915 \mathrm{mg} / \mathrm{kg}$ ), magnésio (1348 a $3316 \mathrm{mg} / \mathrm{kg}$ ) e ferro (141 a $220 \mathrm{mg} / \mathrm{kg})$. Não tendo sido detectado a presença de cobre nas folhas procedentes do horto da EMBRAPA e nem de zinco nas folhas procedentes do horto da EMBRAPA e feira do Ver-o-peso. Tem-se conhecimento que essa planta é usada empiricamente para o tratamento de anemia causada pela deficiência de ferro. As análises dos chás de pariri, observados na Tabela 3 , mostraram que boa parte do cálcio (6955 a $20058 \mathrm{mg} / \mathrm{L}$ ), magnésio (2390 a $3094 \mathrm{mg} / \mathrm{L}$ ) e ferro (40 a $61 \mathrm{mg} / \mathrm{L}$ ) encontramse disponíveis para extração nos chás. Porém, não foram detectadas a presença de cobre nas amostras procedentes dos hortos da EMBRAPA e ALBRAS e zinco nas três localidades.

O sucurijú foi a espécie que se apresentou com maior teor nos minerais analisados. Na Tabela 2, observase que as folhas apresentaram concentrações entre 21619 a $31736 \mathrm{mg} / \mathrm{kg}$ para cálcio, 4783 a $11900 \mathrm{mg} / \mathrm{kg}$ para magnésio, 127 a $141 \mathrm{mg} / \mathrm{kg}$ para ferro, 19 a $56 \mathrm{mg} / \mathrm{kg}$ para cobre e de 31 a $100 \mathrm{mg} / \mathrm{kg}$ para zinco. Os chás de sucurijú mostraram que os minerais analisados encontram-se ligados a espécies químicas facilmentes dissolvidas em água quente. Na Tabela 3, observa-se que os chás dessa planta apresentaram consideráveis índices de cálcio (17722 a $22336 \mathrm{mg} / \mathrm{L}$ ), magnésio (4531 a $9370 \mathrm{mg} / \mathrm{L}$ ), ferro (20 a $87 \mathrm{mg} / \mathrm{L})$, cobre (7 a $16 \mathrm{mg} / \mathrm{L})$ e zinco (9 a 41 $\mathrm{mg} / \mathrm{L})$.

O elixir-paregórico, apesar de apresentar elevado teor de cálcio nas folhas (18273 a $21417 \mathrm{mg} / \mathrm{kg}$ ), mostrou concentrações relativamente baixas nos chás (2036 a 4344 $\mathrm{mg} / \mathrm{L})$ mostrando que a maior parte desse elemento está pouco disponível para extração aquosa. Para magnésio foram verificadas concentrações entre 2737 a $5529 \mathrm{mg} / \mathrm{kg}$ nas folhas e 618 a $4023 \mathrm{mg} / \mathrm{L}$ nos chás. Para o ferro foram observados teores entre 103 a $190 \mathrm{mg} / \mathrm{kg}$ nas folhas e $39 \mathrm{a}$ $60 \mathrm{mg} / \mathrm{L}$ nos chás. Não foi detectado a presença de cobre e zinco nos chás de elixir paregórico (Tabelas 2 e 3).

As concentrações dos minerais analisados, observadas nas Tabelas 2 e 3 apresentaram concentrações bastante variáveis, considerando-se as mesmas espécies coletadas em diferentes locais. Segundo Hewitt \& Smith (1975) e Malavolta (1980) essa variação é devido a inúmeros fatores como composição mineralógica do solo, clima, acidez, matéria orgânica, umidade do solo e adubação. Observou-se, ainda, que de uma forma geral as plantas das espécies elixir paregórico e pariri obtiveram maior conteúdo de $\mathrm{Ca}, \mathrm{Mg}, \mathrm{Fe}, \mathrm{Cu}$ e $\mathrm{Zn}$ nos solos tratados com caroços de açaí e resto de alimentos, utilizados no horto da ALBRAS. Já as plantas da espécie sucurijú 
mostraram maior teor de $\mathrm{Ca}, \mathrm{Mg}, \mathrm{Cu}$ e $\mathrm{Zn}$ nos solos tratados com esterco bovino e restos de plantas, utilizado no horto EMBRAPA. Apesar de não termos informações sobre o tratamento do solo das plantas adquiridas na feira do Ver-o-peso, não foi encontrado indícios que indiquem contaminação das amostras com relação aos metais analisados.

Observando-se os teores dos minerais nos chás das plantas analisadas (Tabela 3) verifica-se que os valores encontrados para cálcio (2036 a $22336 \mathrm{mg} / \mathrm{L}$ ) e magnésio (618 a 9370 mg/L) são mais expressivos que os encontrados em chás de plantas como anador, capim-santo, cidreira, colônia, eparema, hortelã-rasteira, malvariço, malva-santa, mastruço e mentrasto, que apresentaram, em média, concentrações entre 110 a $3160 \mathrm{mg} / \mathrm{L}$ para cálcio e 230 a 1100 mg/L para magnésio (Almeida et al., 2002).

Andrade et al., (2005) determinaram o conteúdo de cobre, ferro e zinco em folhas de varias espécies de plantas medicinais como boldo, carqueja, catuaba, ervasão-joão, espinheira-santa, guaraná, jurubeba, pata-devaca e sene, as quais, apresentaram concentrações entre 7,30 a $14,40 \mathrm{mg} / \mathrm{L}$ para cobre e 22,50 a $57,0 \mathrm{mg} / \mathrm{L}$ para ferro. Observa-se na Tabela 3 que as espécies estudadas apresentaram valores de ferro entre 20 a $87 \mathrm{mg} / \mathrm{L}$ e na espécie sucurijú verificaram-se concentrações de cobre entre 7 a $16 \mathrm{mg} / \mathrm{L}$, valores estes superiores aos detectados pelo autor.

\section{Comparação dos teores de minerais nos chás com os valores recomendados pela OMS}

Analisando-se os resultados da Tabelas 3 e comparando-se com os valores de necessidades diárias de minerais recomendadas pela (OMS) Organização Mundial da Saúde, que indica para suprir às necessidades diárias de uma pessoa adulta a quantidade média de $450 \mathrm{mg}$ de cálcio, $300 \mathrm{mg}$ de magnésio, 12,5 mg de zinco e 3,0 mg de cobre, além de recomendar $10 \mathrm{mg}$ de ferro para o homem e $20 \mathrm{mg}$ de ferro para a mulher. É possível, então, calcular com a fórmula abaixo o volume de chá que corresponderia à metade do que é recomendado para ingestão diária de uma pessoa adulta (Tabela 4), onde os chás das plantas poderiam contribuir para a complementação das dietas alimentares, principalmente de cálcio, magnésio e ferro.

$$
\mathrm{V}_{(\mathrm{mL})}=\mathrm{B} \times 1000 / \mathrm{A}
$$

Onde,

$\mathrm{A}=$ teor do elemento no chá $(\mathrm{mg} / \mathrm{L})$

$\mathrm{B}=$ metade do indicado para o consumo diário do elemento, segundo a OMS.

A determinação de minerais nas plantas é bastante importante, no entanto, uma caracterização também da parte orgânica faz-se necessário para uma possível indicação alternativa de sais minerais.

Tabela 2. Teores de minerais nas folhas de pariri, sucurijú e elixir paregórico $(\mathrm{mg} / \mathrm{kg})$

\begin{tabular}{lccccc}
\hline Amostra/folha & Cálcio & Magnésio & Ferro & Cobre & Zinco \\
\hline Pariri-ALBRAS & $20915,97 \pm 141,80$ & $3170,70 \pm 137,69$ & $220,75 \pm 3,34$ & $14,70 \pm 1,62$ & $40,02 \pm 1,16$ \\
Pariri-EMBRAPA & $15045,06 \pm 240,72$ & $3316,72 \pm 147,53$ & $141,36 \pm 2,93$ & ND $^{1}$ & ND $^{2}$ \\
Pariri-Ver-o-peso & $19535,66 \pm 138,55$ & $1348,07 \pm 154,46$ & $169,40 \pm 5,15$ & $12,80 \pm 1,82$ & ND $^{2}$ \\
Sucurijú-ALBRAS & $21619,88 \pm 255,90$ & $6228,21 \pm 142,47$ & $141,40 \pm 4,15$ & $24,50 \pm 1,75$ & $31,46 \pm 2,18$ \\
Sucurijú-EMBRAPA & $26751,21 \pm 226,25$ & $11900,14 \pm 174,65$ & $131,25 \pm 1,80$ & $56,92 \pm 2,43$ & $100,07 \pm 1,48$ \\
Sucurijú-Ver-o-peso & $31736,69 \pm 167,54$ & $4783,00 \pm 177,75$ & $127,21 \pm 4,26$ & $19,44 \pm 1,34$ & $42,36 \pm 2,82$ \\
Elixir p.-ALBRAS & $21417,82 \pm 248,90$ & $5529,06 \pm 253,15$ & $190,14 \pm 2,16$ & $8,01 \pm 1,63$ & $30,30 \pm 1,87$ \\
Elixir p.-EMBRAPA & $18273,85 \pm 252,30$ & $5300,23 \pm 163,90$ & $103,02 \pm 3,90$ & $\mathrm{ND}^{1}$ & $\mathrm{ND}^{2}$ \\
Elixir p.-Ver-o-peso & $20882,78 \pm 233,00$ & $2737,84 \pm 250,20$ & $121,80 \pm 2,95$ & $7,27 \pm 2,62$ & $\mathrm{ND}^{2}$ \\
\hline
\end{tabular}

$\mathrm{ND}^{1}$ : Não detectado $(<0,040 \mathrm{mg} / \mathrm{L}), \mathrm{ND}^{2}$ : Não detectado $(<0,020 \mathrm{mg} / \mathrm{L})$

Tabela 3. Teores de minerais nos chás de pariri, sucurijú e elixir paregórico (mg/L)

\begin{tabular}{|c|c|c|c|c|c|}
\hline Amostra/chá & Cálcio & Magnésio & Ferro & Cobre & Zinco \\
\hline Pariri-ALBRAS & $20058,84 \pm 126,30$ & $3094,42 \pm 106,80$ & $40,36 \pm 3,03$ & $\mathrm{ND}^{1}$ & $\mathrm{ND}^{2}$ \\
\hline Pariri-EMBRAPA & $6955,50 \pm 146,00$ & $2390,54 \pm 140,02$ & $50,69 \pm 1,46$ & $\mathrm{ND}^{1}$ & $\mathrm{ND}^{2}$ \\
\hline Pariri-Ver-o-peso & $12671,13 \pm 197,08$ & $1019,38 \pm 160,02$ & $61,94 \pm 4,08$ & $10,26 \pm 0,35$ & $\mathrm{ND}^{2}$ \\
\hline Sucurijú-ALBRAS & $18274,52 \pm 135,00$ & $4783,26 \pm 124,21$ & $38,72 \pm 2,16$ & $7,62 \pm 1,57$ & $9,52 \pm 0,81$ \\
\hline Sucurijú-EMBRAPA & $17722,13 \pm 229,10$ & $9370,49 \pm 146,52$ & $20,31 \pm 2,48$ & $16,64 \pm 1,71$ & $41,79 \pm 1,96$ \\
\hline Sucurijú-Ver-o-peso & $22336,90 \pm 174,60$ & $4531,11 \pm 139,20$ & $87,75 \pm 2,97$ & $13,80 \pm 1,27$ & $19,34 \pm 0,75$ \\
\hline Elixir p.-ALBRAS & $4344,68 \pm 143,51$ & $4023,65 \pm 247,31$ & $39,54 \pm 3,92$ & $\mathrm{ND}^{1}$ & $\mathrm{ND}^{2}$ \\
\hline Elixir p.-EMBRAPA & $3210,81 \pm 195,20$ & $2622,06 \pm 143,00$ & $60,62 \pm 1,88$ & $\mathrm{ND}^{1}$ & $\mathrm{ND}^{2}$ \\
\hline Elixir p.-Ver-o-peso & $2036,52 \pm 136,00$ & $618,03 \pm 127,30$ & $60,23 \pm 2,97$ & $\mathrm{ND}^{1}$ & $\mathrm{ND}^{2}$ \\
\hline
\end{tabular}

$\mathrm{ND}^{1}$ : Não detectado $(<0,040 \mathrm{mg} / \mathrm{L}), \mathrm{ND}^{2}$ : Não detectado $(<0,020 \mathrm{mg} / \mathrm{L})$ 
Tabela 4. Volume de chá que corresponde metade do que é recomendado para ingestão diária de minerais, segundo a OMS

\begin{tabular}{|c|c|c|c|c|c|c|}
\hline \multirow{2}{*}{ Chá } & \multirow[t]{2}{*}{$\mathrm{Ca}(\mathrm{mL})$} & \multirow[t]{2}{*}{$\mathrm{Mg}(\mathrm{mL})$} & \multicolumn{2}{|c|}{$\mathrm{Fe}(\mathrm{mL})$} & \multirow[t]{2}{*}{$\mathrm{Cu}(\mathrm{mL})$} & \multirow[t]{2}{*}{$\mathrm{Zn}(\mathrm{mL})$} \\
\hline & & & Homem & Mulher & & \\
\hline Pariri / ALBRAS & 11 & 48 & 124 & 248 & \# & \# \\
\hline Pariri / EMBRAPA & 32 & 63 & 99 & 197 & \# & \# \\
\hline Pariri / Ver-o-peso & 18 & 147 & 81 & 161 & 146 & \# \\
\hline Sucurijú /ALBRAS & 12 & 31 & 129 & 258 & 197 & 656 \\
\hline Sucurijú / EMBRAPA & 13 & 16 & 246 & 292 & 90 & 150 \\
\hline Sucurijú / Ver-o-peso & 10 & 33 & 57 & 114 & 109 & 323 \\
\hline Elixir p. / ALBRAS & 52 & 37 & 126 & 253 & \# & \# \\
\hline Elixir p. / EMBRAPA & 70 & 57 & 82 & 165 & \# & \# \\
\hline Elixir p. / Ver-o-peso & 110 & 243 & 83 & 166 & \# & \# \\
\hline
\end{tabular}

\# = indica que não foi evidenciada a presença do metal no chá

\section{REFERÊNCIAS}

Almeida MMB, Lopes MFG, Nogueira CMD, Magalhães CEC, Moraes NMT 2002. Determinação de nutrientes minerais em plantas medicinais. Cienc Tecnol Aliment 22: 94-97.

Andrade ECB, Alves SP, Takase I 2005. Avaliação do uso de ervas medicinais como suplemento nutricional de ferro, cobre e zinco. Cienc Tecnol Aliment 25: 591-596.

Berg MEVDE 1993. Plantas medicinais na Amazônia. BelémPA: Coleção Adolfho Ducke.

Burton BT 1979. Nutrição Humana. São Paulo: Mc Graw-Hill do Brasil.

Delaporte RH, Guzen KP, Takemura OS, Mello JCP 2005. Estudo mineral das espécies vegetais Alternanthera brasiliana (L.) Kuntze e Bouchea fluminensis (Vell) Mold. Rev Bras Farmacogn 15: 133-136.

Duarte RPS, Pasqual A 2000. Avaliação do cádmio (Cd), chumbo $(\mathrm{Pb})$, níquel $(\mathrm{Ni})$ e zinco $(\mathrm{Zn})$ em solos, plantas e cabelos humanos. Energ Agric 15: 46-58.

FAO/WHO 1998. World Health Organization and Food and Agriculture Organization of the United Nations. Vitamin and mineral requirements in human nutrition. Thailand: Bangkok.

Favier A 1991. Les oligoéléments en nutrition humaine. In: CAPPUIS, P. (Ed) Les oligoéléments en medicine et biologic. Paris: Editions médicales Internacionales, p. 41-74.

Flores H, Campos FACS, Silva MBM, Lins MHCB 1998. Enriquecimento de alimentos: presente e futuro. Ciênc Tecnol Aliment 30: 49-55.

Franco F 1998. Tabela de composição de alimentos. São Paulo: Atheneu.

Harper HA, Mayes RA 1982. Manual de Química fisiologica. São Paulo: Atheneu.

Hewitt EJ, Smith TA 1975. Plant mineral nutrition. London: English University Press.

Krause MV, Mahan LK 1991. Alimentos, nutrição e dietoterapia. São Paulo: Livraria Roca.

Lameira OA, Oliveira ECP, Paiva JS, Teixeira LB, Germano
VLC 2004. Plantas Medicinais: Uso e Manipulação. Comunicado Técnico 128. Ministério da Agricultura Pecuária e Abastecimento. Belém-PA, Brasil.

Lehninger AL, Nelson DL, Cox, MM 1998. Princípios de Bioquímica. São Paulo: Sarvier.

Lopes MFG, Almeida MMB, Nogueira CMD, Morais NMT, Magalhães CEC 2002. Estudo mineral de plantas medicinais. Rev Bras Farmacogn 12: 115-116.

Lopes MFG, Morais NMT, Nogueira CMD, Vasconcelos NMS, Silva WC, Gonzaga MLC 1996. Determinações Analíticas em alfavaca, quebra-pedra, sabugueiro, eucalipto e cajáumbu. Anais Assoc Bras Quím 45: 155-157.

Lorenzi HE, Matos FJA 2002. Plantas medicinais no Brasil/ Nativas e exóticas. Nova Odessa, SP: Plantarum.

Machado MVF, Canniatti-Brazaca SG, Piedade SMS 2006. Avaliação da disponibilidade de ferro em ovo, cenoura e couve e em suas misturas. Cienc Tecnol Aliment 26: 610-618.

Maciel MAM, Pinto AC, Veiga Jr VF 2002. Plantas medicinais: a necessidade de estudos multidisciplinares. Quim Nova 25: 429-438.

Malavolta, E 1980. Elementos de nutrição mineral de plantas. São Paulo: Agronômica Ceres.

Martins AG, Rosário DL, Barros MN, Jardim, MAG 2005. Levantamento etnobotânico de plantas medicinais, alimentares e tóxicas da Ilha do Combu, Município de Belém, Estado do Pará, Brasil. Rev Bras Farm 86: 2130 .

Pinto NAVD, Vilas Boas BM, Carvalho VD 1999. Caracterização mineral das folhas de taioba (Xanthosoma sagittifolium Schott). Cienc e Agrotec 23: 57-61.

Silva FC 1999. Manual de Análises Químicas de Solos, Plantas e Fertilizantes. Campinas, SP: Embrapa Informática Agropecuária.

Word Health Organization (WHO) 1996. Trace Elements in Human Nutrition and Health. Geneva. 